\title{
ROBUSTIFICATION OF CHAOS IN 2D MAPS
}

\author{
ZERAOULIA ELHADJ*,‡ and J. C. SPROTT ${ }^{\dagger, \S}$ \\ *Department of Mathematics, \\ University of Tébessa (12002), Algeria \\ ${ }^{\dagger}$ Department of Physics, University of Wisconsin, \\ Madison, WI 53706, USA \\ ${ }^{\ddagger}$ zeraoulia@mail.univ-tebessa.dz \\ ${ }^{\ddagger}$ zelhadj12@yahoo.fr \\ §sprott@physics.wisc.edu \\ Received 10 February 2011 \\ Revised 21 June 2011
}

\begin{abstract}
Robust chaos is defined as the absence of periodic windows and coexisting attractors in some neighborhood of the parameter space since the existence of such windows in the chaotic region implies fragility of the chaos. In this paper, we introduce a new terminology called robustification of chaos, which means creating robust chaos (in the sense of the above definition) in a dynamical system. As a first step, a new chaotification (robustification) method to generate robust chaos in planar maps is presented using simple piecewise smooth feedback to create a border collision bifurcation in the resulting system under some realizable conditions. The results are applied to an elementary example to illustrate the validity of the proposed method.
\end{abstract}

Keywords: Robustification of chaos; chaotification; planar discrete mapping; piecewise smooth feedback controller; homoclinic chaos.

PACS Number(s): 05.45.-a, 05.45.Gg

\section{Introduction}

Chaos is the idea that a system will produce very different long-term behaviors when the initial conditions are perturbed only slightly. Anticontrol of chaos is a technique used to create or enhance the system complexity for some novel, timeor energy-critical interdisciplinary application. Examples include high-performance circuits and devices, liquid mixing, chemical reactions, biological systems, crisis management, secure information processing, and critical decision-making in politics, economics, as well as military applications, etc. There are several works that focus on this topic. In particular, chaos and bifurcation theories are the main tools used in analyzing anticontrol of chaos, and it can be implemented by the suitable design of controllers, devices, and circuitry.

Robust chaos is defined by the absence of periodic windows and coexisting attractors in some neighborhood of the parameter space. The existence of these 
windows in some chaotic regions means that small changes of the parameters would destroy the chaos, implying the fragility of this type of chaos. The notion of robust chaos was first discussed in the early 1992 by Majumdar and Mitra in [28] where they identify a quadratic family of dynamic optimization models and they prove that the resulting chaos is robust in the above sense. A very same phenomenon related to electronics was subsequently published with the very same name in [29]. An overview of some important issues concerning the robustness of chaos in dynamical systems and their applications to the real world were given in [12].

There are many practical applications that require robust chaos $[12,28,29]$ such as in communications and spreading the spectrum of switched-mode power supplies to avoid electromagnetic interference $[10,11]$ where it is necessary to obtain reliable operation in the chaotic mode, and thus robust chaos is required. Practical examples of demonstrating robust chaos can be found in electrical engineering as shown in $[12,13,21]$. The most important results about robust chaos in dynamical systems and its real applications can be found in [3] along with many elementary examples and conjectures. In particular, robust chaos is an essential feature resulting from the border collision bifurcations observed in piecewise systems [12, 13, 21]. In fact, many physical and engineering systems have been found that are governed by a class of continuous or discontinuous maps $[12,13,21]$ where the discrete-time state space is divided into two or more compartments with different functional forms of the map separated by borderlines $[12,13,21]$. On the other hand, many chaotification methods have been proposed to create or enhance the system complexity for novel, time- or energy-critical interdisciplinary applications. Examples include high-performance circuits and devices, liquid mixing, chemical reactions, biological systems, crisis management, secure information processing, and critical decision-making in politics, economics, and military applications. In other words, anticontrolling chaos produces chaotic behavior in a system that would not otherwise be chaotic $[1,2,6-9,16-20]$. For example, these chaotification schemes were presented for discrete mappings using Lyapunov exponents, or by the use of several modified versions of the Marotto theorem [4,5,9-15], or by the use of the Li-Yorke definition of chaos [14].

Some recent works on robust chaos can be found in [23-25]. Indeed in [23], the critical behavior of the Lyapunov exponent near the transition to robust chaos via type-III intermittency was determined for a $1 \mathrm{D}$ singular maps. The critical boundaries separating the region of robust chaos was calculated along a critical exponent (as a function of the order of the singularity of the map) expressing the scaling of the Lyapunov exponent along the critical curve corresponding to the type-III intermittency. This result contrasts with the well-known predictions for the scaling behavior of the Lyapunov exponent in type-III intermittency. In [24], an extension of several proposed methods to construct $1 \mathrm{D}$ chaotic maps $[26,27]$ with exactly known natural invariant measure. The first method was proposed for the construction of maps with robust chaos and prescribed invariant measure and constant Lyapunov exponent. The second method was obtained by relaxing 
one condition in the approach described in $[26,27]$ to construct robust chaos with prescribed constant invariant measure and varying Lyapunov exponent. The third method was based on an extension of a condition in $[26,27]$ and provides a new method to construct robust chaos with exactly computed invariant measure and known varying Lyapunov exponent. The fourth method was based on the use of diffeomorphisms to construct maps with robust chaos with any number of parameters and prescribed invariant measure and Lyapunov exponent. An example of robust chaos in continuous time systems can be found in [25]. Indeed, a description of the regular self-organization observed numerically in natural phenomena modeled by differential equations was presented. A classification of periodic and chaotic (robust) behaviors was given in several regions of bifurcation parameters of a two Rôssler's and a chemical oscillators.

The essential motivation of the present work is to create robust chaos in general $2 \mathrm{D}$ discrete mappings via the controller of a simple piecewise smooth function under some realizable conditions.

\section{Robustifying 2D Maps Using a Piecewise Smooth Feedback Controller}

In this section, we present our method for robustifying chaos in $2 \mathrm{D}$ mappings. For this purpose, consider the general two-dimensional map (not necessarily chaotic) of the form $f(x, y, \rho)=\left(f_{1}(x, y, \rho), f_{2}(x, y, \rho)\right)$ where the function $f$ is assumed to be locally continuously differentiable and depends on a single parameter $\rho$. The goal of the control is to create a border collision bifurcation leading to robust chaos in the simplest way, i.e. by considering the controlled map given by

$$
g(x, y, \rho)=\left(\begin{array}{l}
g_{1}(x, y, \rho)=f_{1}(x, y, \rho)+\alpha|x| \\
g_{2}(x, y, \rho)=f_{2}(x, y, \rho)+\beta|x|
\end{array}\right),
$$

where $\alpha$ and $\beta$ are real bifurcation parameters to be determined. The line $x=0$ divides the phase plane into two regions $R_{1}=\left\{(x, y) \in \mathbb{R}^{2}, x<0\right\}$ and $R_{2}=$ $\left\{(x, y) \in \mathbb{R}^{2}, x>0\right\}$. The controlled map (1) is a piecewise smooth map since the controller $u(x, y)=(\alpha|x|, \beta|x|)$ is also smooth. It is also clear that the functions $f_{1}$ and $f_{2}$ are both continuous and have continuous derivatives, and thus the controlled map $g$ is continuous, but its derivative is discontinuous at the borderline $x=0$. Furthermore, it is clear that the one-sided partial derivatives at the border are finite, and in each subregion $R_{1}$ and $R_{2}$, the controlled map (1) has only two fixed points $P_{1}$ and $P_{1}$ for values $\rho_{*}$ of the parameter $\rho$ by assuming that the equations $f_{1}(x, y, \rho)+\alpha|x|=x$ and $f_{2}(x, y, \rho)+\beta|x|=y$ have only one root $P_{1}$ and $P_{2}$, respectively, for some ranges of the parameters $\rho, \alpha$ and $\beta$. The control design $u(x, y)$ leads to systems of linear inequalities for the unknown parameters $\alpha$ and $\beta$ in terms of the sets $\left(\beta_{j}\right)_{1 \leq i \leq 4}$ and $\left(\gamma_{j}\right)_{1 \leq i \leq 4}$ assumed to be known and defined by $\frac{\partial f_{1}(x, y, \rho)}{\partial x}\left(P_{1}\right)=\beta_{1}, \frac{\partial f_{1}(x, y, \rho)}{\partial y}\left(P_{1}\right)=\beta_{2}, \frac{\partial f_{2}(x, y, \rho)}{\partial x}\left(P_{1}\right)=\beta_{3}, \frac{\partial f_{2}(x, y, \rho)}{\partial y}\left(P_{1}\right)=\beta_{4}$, $\frac{\partial f_{1}(x, y, \rho)}{\partial x}\left(P_{2}\right)=\gamma_{1}, \frac{\partial f_{1}(x, y, \rho)}{\partial y}\left(P_{2}\right)=\gamma_{2}, \frac{\partial f_{2}(x, y, \rho)}{\partial x}\left(P_{1}\right)=\gamma_{3}$ and $\frac{\partial f_{2}(x, y, \rho)}{\partial y}\left(P_{1}\right)=\gamma_{4}$. 
It was shown in [13] that the normal form of the controlled map (1) is given by

$$
N(x, y)=\left\{\begin{array}{ll}
\left(\begin{array}{cc}
\tau_{1} & 1 \\
-\delta_{1} & 0
\end{array}\right)\left(\begin{array}{l}
x \\
y
\end{array}\right)+\left(\begin{array}{l}
1 \\
0
\end{array}\right) \mu, & \text { if } x<0 \\
\left(\begin{array}{cc}
\tau_{2} & 1 \\
-\delta_{2} & 0
\end{array}\right)\left(\begin{array}{l}
x \\
y
\end{array}\right)+\left(\begin{array}{l}
1 \\
0
\end{array}\right) \mu, & \text { if } x>0
\end{array},\right.
$$

where $\mu$ is the new bifurcation parameter of the map (2). The normal form (2) has two fixed points $P_{L}=\left(\frac{\mu}{1-\tau_{1}+\delta_{1}}, \frac{-\delta_{1} \mu}{1-\tau_{1}+\delta_{1}}\right) \in R_{1}$ and $P_{R}=\left(\frac{\mu}{1-\tau_{2}+\delta_{2}}, \frac{-\delta_{2} \mu}{1-\tau_{2}+\delta_{2}}\right) \in R_{2}$ (with eigenvalues $\lambda_{1,2}$ and $\omega_{1,2}$, respectively). The stability of these fixed points is determined by the eigenvalues of the corresponding Jacobian matrix, i.e. $\lambda=\frac{1}{2}(\tau \pm$ $\sqrt{\tau^{2}-4 \delta}$ ). Here $\mu$ is a parameter and $\tau_{i}, \delta_{i}, i=1,2$ are the traces and determinants of the corresponding matrices of the linearized map in the two subregions $R_{1}$ and $R_{2}$ evaluated at $P_{1}$ and $P_{2}$, respectively, that is $A_{1}=\left(\begin{array}{ll}\beta_{1} & \beta_{2} \\ \beta_{3} & \beta_{4}\end{array}\right)+\left(\begin{array}{cc}-\alpha & 0 \\ -\beta & 0\end{array}\right)$ and $A_{2}=\left(\begin{array}{ll}\gamma_{1} & \gamma_{2} \\ \gamma_{3} & \gamma_{4}\end{array}\right)+\left(\begin{array}{ll}\alpha & 0 \\ \beta & 0\end{array}\right)$. In the case of the controlled map (1), the values of $\tau_{i}, \delta_{i}$, $i=1,2$ are given by $\tau_{1}=-\alpha+\beta_{1}+\beta_{4}, \tau_{2}=\alpha+\gamma_{1}+\gamma_{4}, \delta_{1}=\beta \beta_{2}-\alpha \beta_{4}+\beta_{1} \beta_{4}-\beta_{2} \beta_{3}$, and $\delta_{2}=\alpha \gamma_{4}-\beta \gamma_{2}+\gamma_{1} \gamma_{4}-\gamma_{2} \gamma_{3}$. It was shown in [12] that robust homoclinic chaos (a kind of persistent chaos in low dimensions [22]) occurs in the piecewise smooth map of the form (2) if one of the following conditions holds:

(a)

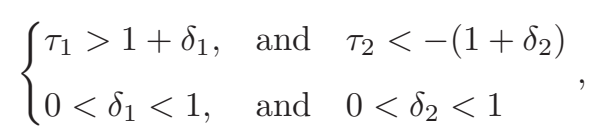

where the parameter range for a boundary crisis is given by

$$
C_{1}: \delta_{1} \tau_{2} \lambda_{1}-\delta_{2} \lambda_{1} \lambda_{2}+\delta_{2} \lambda_{2}-\delta_{1} \tau_{2}+\delta_{1} \tau_{1}-\delta_{1}-\lambda_{1} \delta_{1}>0
$$

Here, inequality (4) determines the condition for stability of the chaotic attractor of the map (1). The robust chaotic orbit persists as $\tau_{1}$ is reduced below $1+\delta_{1}$.

(b)

$$
\left\{\begin{array}{l}
\tau_{1}>1+\delta_{1}, \quad \text { and } \quad t_{2}<-\left(1+\delta_{2}\right) \\
\delta_{1}<0, \quad \text { and } \quad-1<\delta_{2}<0 \\
C_{2}: \frac{\lambda_{1 L}-1}{\tau_{1}-1-\delta_{1}}>\frac{\lambda_{2 R}-1}{\tau_{2}-1-\delta_{2}}
\end{array} .\right.
$$

The condition for stability of the chaotic attractor is also determined by (4). However, if the third condition of (5) is not satisfied, then the condition for the existence of a chaotic attractor changes to

$$
C_{3}: \frac{\omega_{2}-1}{\tau_{2}-1-\delta_{1}}<\frac{\left(\tau_{1}-\delta_{1}-\lambda_{2}\right)}{\left(\tau_{1}-1-\delta_{1}\right)\left(\lambda_{2}-\tau_{2}\right)} .
$$


(c) The remaining ranges for the quantity $\tau_{1,2}, \delta_{1,2}$ can be determined in some cases using the same logic as in the above two cases, or there is no analytic condition for a boundary crisis, and it has to be determined numerically. Thus, from the above three cases, we shall show that for certain values of $\alpha$ and $\beta$, the controlled map (1) has robust homoclinic chaos, where the values $\left(\beta_{i}\right)_{1 \leq i \leq 4}$ and $\left(\gamma_{i}\right)_{1 \leq i \leq 4}$ are assumed to be known. Indeed, for the first case (a), condition (3) gives the following inequalities:

$$
\left\{\begin{array}{l}
\phi_{1}<\beta_{2} \beta<\min \left\{\phi_{2}, \phi_{3}\right\} \\
\max \left\{\phi_{4}, \phi_{5}\right\}<\gamma_{2} \beta<\phi_{6}
\end{array}\right.
$$

where $\phi_{1}=\alpha \beta_{4}-\beta_{1} \beta_{4}+\beta_{2} \beta_{3}, \phi_{2}=\beta_{1}-\alpha+\beta_{4}+\alpha \beta_{4}-\beta_{1} \beta_{4}+\beta_{2} \beta_{3}-1, \phi_{3}=$ $\alpha \beta_{4}-\beta_{1} \beta_{4}+\beta_{2} \beta_{3}+1, \phi_{4}=\alpha+\gamma_{1}+\gamma_{4}+\alpha \gamma_{4}+\gamma_{1} \gamma_{4}-\gamma_{2} \gamma_{3}+1, \phi_{5}=\alpha \gamma_{4}+$ $\gamma_{1} \gamma_{4}-\gamma_{2} \gamma_{3}-1$, and $\phi_{6}=\alpha \gamma_{4}+\gamma_{1} \gamma_{4}-\gamma_{2} \gamma_{3}$. The two inequalities in (7) hold if and only if $\phi_{1}<\phi_{2}, \phi_{1}<\phi_{3}, \phi_{4}<\phi_{6}$, and $\phi_{5}<\phi_{6}$, that is,

$$
\alpha<\min \left\{\beta_{1}+\beta_{4}-1,-\gamma_{1}-\gamma_{4}-1\right\} .
$$

The stability of the resulting homoclinic chaos can be tested using inequality (4). In fact, it is difficult to solve rigorously for this stability condition (or the conditions for the two remaining cases (b) and (c) above) due to the presence of complicated square formulas, but one can use numerical estimates. From (7), it is clear that the value of the parameter $\beta$ depends mainly on the signs of $\beta_{2}$ and $\gamma_{2}$. If $\beta_{2}>0$ and $\gamma_{2}>0$, then (7) becomes

$$
\left\{\begin{array}{l}
\frac{\phi_{1}}{\beta_{2}}<\beta<\frac{\min \left\{\phi_{2}, \phi_{3}\right\}}{\beta_{2}} \\
\frac{\max \left\{\phi_{4}, \phi_{5}\right\}}{\gamma_{2}}<\beta<\frac{\phi_{6}}{\gamma_{2}}
\end{array} .\right.
$$

If $\beta_{2}<0$ and $\gamma_{2}<0$, then (7) becomes

$$
\left\{\begin{array}{l}
\frac{\phi_{1}}{\beta_{2}}>\beta>\frac{\min \left\{\phi_{2}, \phi_{3}\right\}}{\beta_{2}} \\
\frac{\max \left\{\phi_{4}, \phi_{5}\right\}}{\gamma_{2}}>\beta>\frac{\phi_{6}}{\gamma_{2}}
\end{array} .\right.
$$

If $\beta_{2}>0$ and $\gamma_{2}<0$, then (7) becomes

$$
\left\{\begin{array}{l}
\frac{\phi_{1}}{\beta_{2}}<\beta<\frac{\min \left\{\phi_{2}, \phi_{3}\right\}}{\beta_{2}} \\
\frac{\max \left\{\phi_{4}, \phi_{5}\right\}}{\gamma_{2}}>\beta>\frac{\phi_{6}}{\gamma_{2}}
\end{array},\right.
$$

that is,

$$
\max \left\{\frac{\phi_{1}}{\beta_{2}}, \frac{\phi_{6}}{\gamma_{2}}\right\}<\beta<\min \left\{\frac{\min \left\{\phi_{2}, \phi_{3}\right\}}{\beta_{2}}, \frac{\max \left\{\phi_{4}, \phi_{5}\right\}}{\gamma_{2}}\right\}
$$


If $\beta_{2}<0$ and $\gamma_{2}>0$, then (7) becomes

$$
\left\{\begin{array}{l}
\frac{\phi_{1}}{\beta_{2}}>\beta>\frac{\min \left\{\phi_{2}, \phi_{3}\right\}}{\beta_{2}} \\
\frac{\max \left\{\phi_{4}, \phi_{5}\right\}}{\gamma_{2}}<\beta<\frac{\phi_{6}}{\gamma_{2}}
\end{array},\right.
$$

that is,

$$
\max \left\{\frac{\min \left\{\phi_{2}, \phi_{3}\right\}}{\beta_{2}}, \frac{\max \left\{\phi_{4}, \phi_{5}\right\}}{\gamma_{2}}\right\}<\beta<\min \left\{\frac{\phi_{1}}{\beta_{2}}, \frac{\phi_{6}}{\gamma_{2}}\right\} .
$$

If $\beta_{2}=0$ and $\gamma_{2}>0$, then (7) becomes

$$
\left\{\begin{array}{l}
\phi_{1}<0<\min \left\{\phi_{2}, \phi_{3}\right\} \\
\frac{\max \left\{\phi_{4}, \phi_{5}\right\}}{\gamma_{2}}<\beta<\frac{\phi_{6}}{\gamma_{2}}
\end{array}\right.
$$

The remaining cases (b) and (c) are similar.

\section{Example}

This paper proposes a chaotification (robustification) algorithm for $2 \mathrm{D}$ discrete mappings, which is a topic that has been extensively studied over the last decade as indicated by $[1,2,6-9,16-20]$ and references therein. The significance of our work relative to the existing literature is that we present a new method for robustifying chaos in two-dimensional discrete maps based on the construction of a simple piecewise smooth feedback controller. In addition, the main assumptions here are not complicated, which makes the result practical and attractive. As an example illustrating the conditions and conclusions in the proposed method, consider the following 2D generalized Hénon mapping:

$$
f(x, y)=\left(\begin{array}{c}
1-1.4(1-\rho) x^{2}+y \\
0.3 x
\end{array}\right) \text {. }
$$

To verify the conditions of the previous method, assume that $\alpha=-1.4 \rho$ and $\beta=0$. Thus the controlled version of the map (16) is given by

$$
f(x, y)=\left(\begin{array}{c}
1-1.4(1-\rho) x^{2}+y-1.4 \rho|x| \\
0.3 x
\end{array}\right) .
$$

It is easy to check numerically that the controlled map (10) satisfies the conditions $C_{2}$ and $C_{3}$ given by (5) and (6), respectively. Indeed, consider the critical curves 
corresponding to the conditions (4), (5), and (6) as follows:

$$
\left\{\begin{array}{l}
C_{1}: \frac{\lambda_{1}-1}{\tau_{1}-1-\delta_{1}}-\frac{\omega_{2}-1}{\tau_{1}-1-\delta_{2}}=0 \\
C_{2}:\left(\tau_{2}-\lambda_{2}\right) \lambda_{1}+\tau_{1}-\tau_{2}-\delta_{1}=0 \\
C_{3}: \frac{\omega_{2}-1}{\tau_{2}-1-\delta_{2}}-\frac{\delta_{1}\left(\tau_{1}-\delta_{1}-\lambda_{2}\right)}{\left(\tau_{1}-1-\delta_{1}\right)\left(\delta_{2} \lambda_{2}-\delta_{1} \tau_{2}\right)}=0
\end{array} .\right.
$$

Applying Newton's method for finding roots of an algebraic equation with an error of $10^{-6}$ to system (18), then Fig. 1 shows that the curve $\left(C_{2}\right)$ has an intersection with the $\rho=0$ axis at $\rho=0.0866592234$, which means that conditions (5) hold for $\rho \in[0,0.0866592234]$, while the curve $\left(C_{1}\right)$ does not intersect the $\rho=0$ axis, then conditions (4) do not hold for all $\rho$, and the curve $\left(C_{3}\right)$ intersects the $\rho=0$ axis also once at $\rho=0.493122734$, then condition (6) holds when $\rho \in[0.493122734,1]$. We note that this property is absent for $\rho=1$.

Thus the controlled map (17) displays robust (the attractor is unique) homoclinic chaos when $\rho \in[0.493122734,1]$. This result is also verified numerically by computing Lyapunov exponents and the bifurcation diagram as shown in Fig. 2. An example of such a chaotic attractor is shown in Fig. 3.

For $\rho<0.493122734$, the chaos exhibited by the controlled map (17) is not robust in some ranges of the variable $\rho$ because there are numerous small periodic windows as shown in Figs. 4(a) and 4(b), for example the period- 8 window at $\rho=0.025$. The contribution and importance of the method is that parameters can be chosen to produce chaos in the Hénon map (17), and the robustification allows

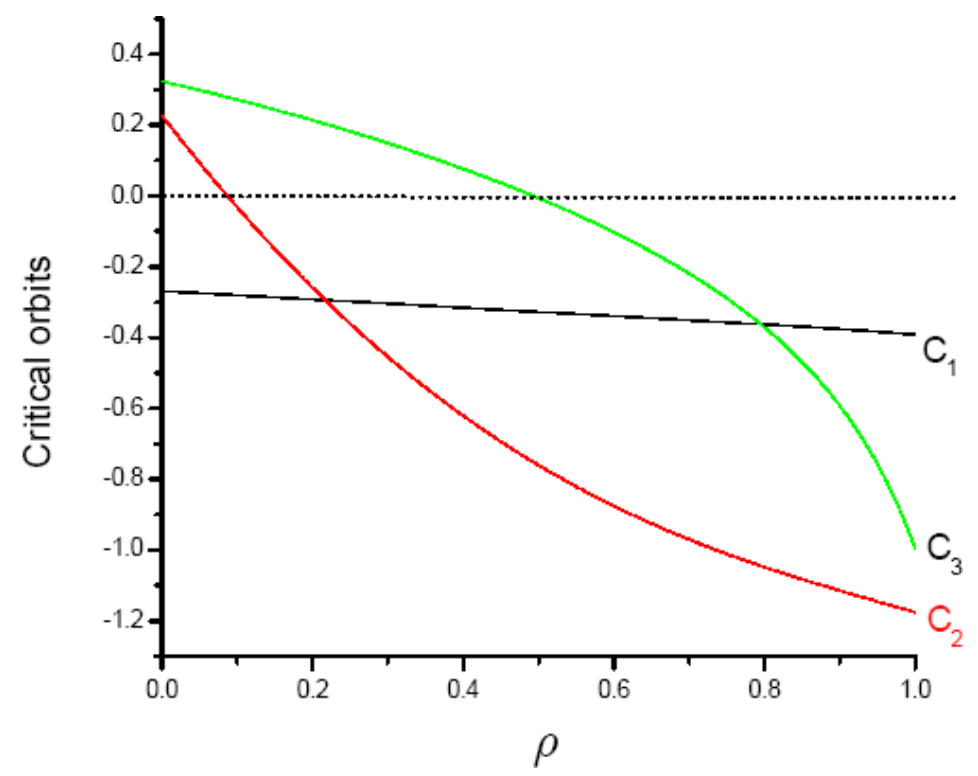

Fig. 1. Critical curves corresponding to the conditions (4), (5) and (6) for the controlled map (17). 
(a)

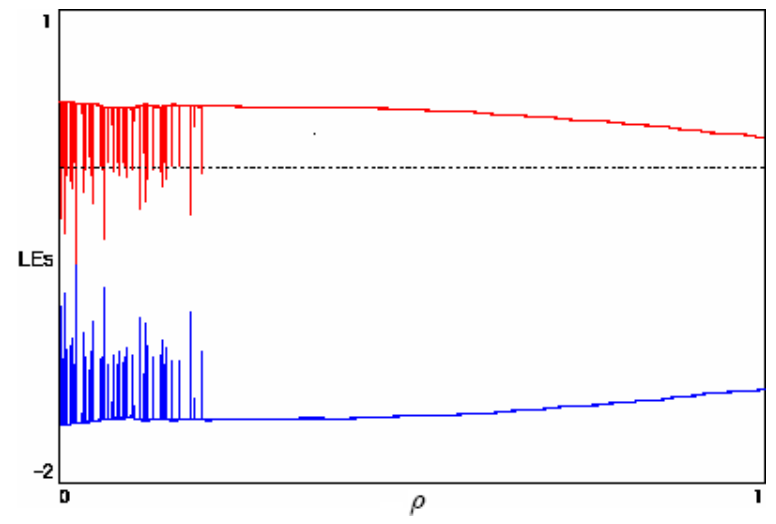

(b)

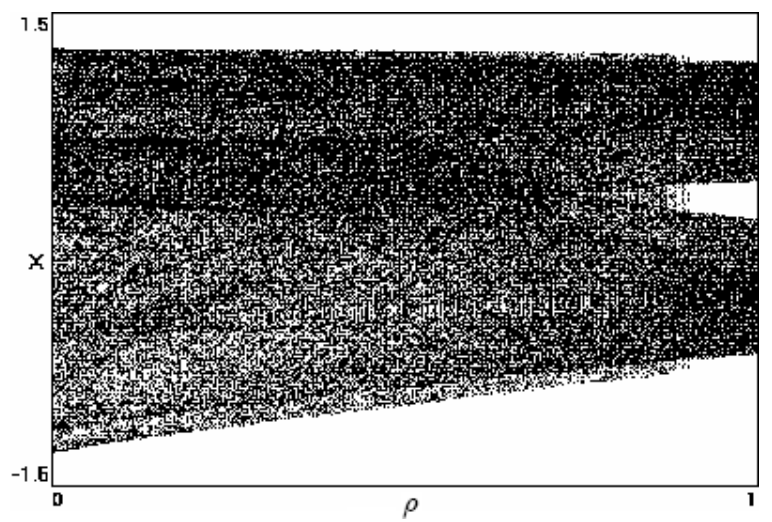

Fig. 2. (a) Variation of the Lyapunov exponents of the controlled map (17) for $0 \leq \rho \leq 1$. (b) The bifurcation diagram of the controlled map (17) for $0 \leq \rho \leq 1$.

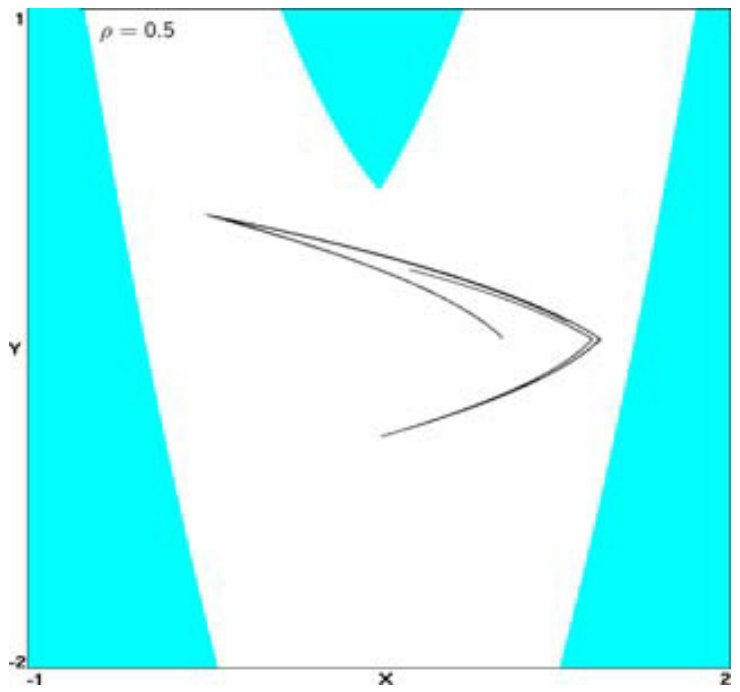

Fig. 3. A robust chaotic attractor with its basin of attraction (in white) obtained from the controlled map (17) for $\rho=0.5$. 


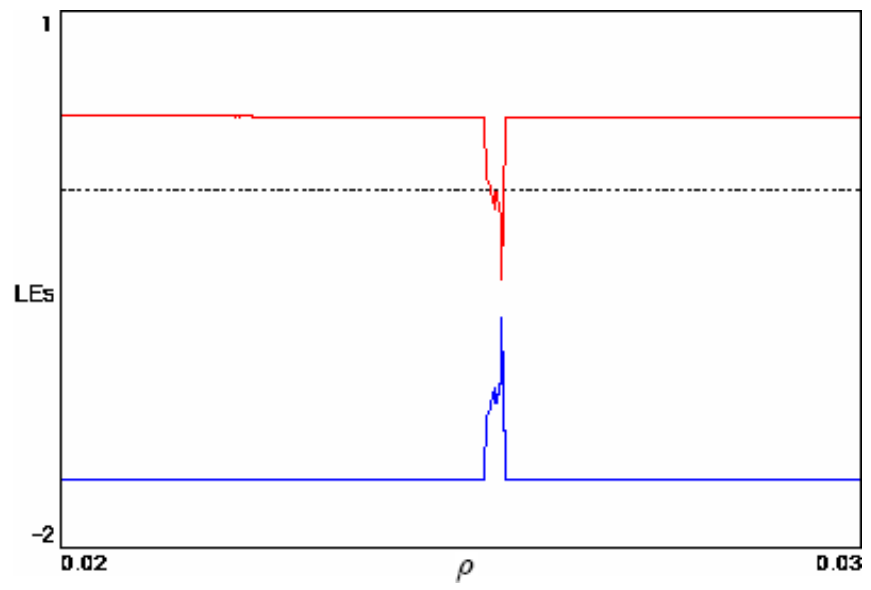

(a)

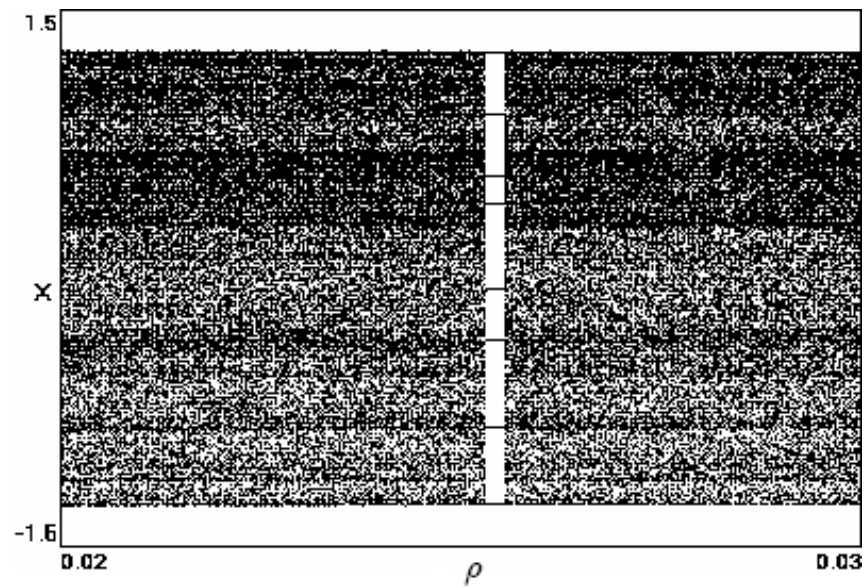

(b)

Fig. 4. (a) Variation of the Lyapunov exponents of the controlled map (17) for $0.02 \leq \rho \leq 0.03$. (b) The bifurcation diagram of the controlled map (17) for $0.02 \leq \rho \leq 0.03$, showing a period-8 attractor obtained for $\rho=0.025$.

one to produce robust chaos over a larger than normal parameter interval as shown in the bifurcation diagrams for map (17).

\section{Conclusion}

We have reported a new chaotification (robustification) method for planar mappings based on a piecewise smooth feedback controller. The significance of the results is discussed, and an example is provided to illustrate the conditions and conclusions of the proposed method. 


\section{References}

[1] Li, C., On super-chaotifying discrete dynamical systems, Chaos Soliton. Fract. 21 (2004) 855-861.

[2] Lai, D. and Chen, G., Making a discrete dynamical system chaotic: Theorical results and numerical simulations, Int. J. Bifur. Chaos 13(11) (2003) 3437-3442.

[3] Zeraoulia, E. and Sprott, J. C., On the robustness of chaos in dynamical systems: Theories and applications, Front. Phys. China 3 (2008) 195-204.

[4] Marotto, F. R., Snap-back repellers imply chaos in $\mathbb{R}^{n}$, J. Math. Anal. Appl. 3 (1978) 199-223.

[5] Marotto, F. R., On redefining a snap-back repeller, Chaos Soliton. Fract. 25 (2005) $25-28$.

[6] Chen, G. and Lai, D., Anticontrol of chaos via feedback, in Proc. of IEEE Conference on Decision and Control, San Diego, CA (1997), pp. 367-372.

[7] Chen, G. and Lai, D., Making a discrete dynamical system chaotic: Feedback control of Lyapunov exponents for discrete-time dynamical system, IEEE Trans. Circ. Syst.-I 44 (1997) 250-253.

[8] Chen, G. and Lai, D., Feedback anticontrol of chaos, Int. J. Bifur. Chaos 8 (1998) $1585-1590$.

[9] Chen, G., Hsu, S. and Zhou, J., Snap-back repellers as a cause of chaotic vibration of the wave equation with a van der Pol boundary condition and energy injection at the middle of the span, J. Math. Phys. 39(12) (1998) 6459-6489.

[10] Ottino, J. M., The Kinematics of Mixing: Stretching, Chaos, and Transport (Cambridge University Press, Cambridge, 1989).

[11] Ottino, J. M., Muzzion, F. J., Tjahjadi, M., Franjione, J. G., Jana, S. C. and Kusch, H. A., Chaos, symmetry, and self-similarity: Exploring order and disorder in mixing processes, Science 257 (1992) 754-760.

[12] Banergee, S., York, J. A. and Grebogi, C., Robust chaos, Phys. Rev. Lett. 80(14) (1998) 3049-3052.

[13] Banerjee, S. and Grebogi, C., Border collision bifurcations in two-dimensional piecewise smooth maps, Phy. Rev. E 59(4) (1999) 4052-4061.

[14] Li, T. Y. and Yorke, J. A., Period three implies chaos, Amer. Math. Monthly 82 (1975) 481-485.

[15] Lin, W., Ruan, J. and Zhou, W., On the mathematical clarification of the snapback repeller in high-dimensional systems and chaos in a discrete neural network model, Int. J. Bifur. Chaos 12(5) (2002) 1129-1139.

[16] Wang, X. F. and Chen, G., On feedback anticontrol of discrete chaos, Int. J. Bifur. Chaos 9 (1999) 1435-1441.

[17] Wang, X. F. and Chen, G., Chaotifying a stable map via smooth small amplitude high-frequency feedback control, Int. J. Circ. Theor. App. 28 (2000) 305-312.

[18] Li, X., Chen, G., Chen, Z. and Yuan, Z., Chaotifying linear Elman networks, IEEE Trans. Neural Netw. 13 (2002) 1193-1199.

[19] Li, Z., Park, J. B., Joo, Y. H., Choi, Y. H. and Chen, G., Anticontrol of chaos for discrete TS fuzzy systems, IEEE Trans. Circuits Syst.-I. 49 (2002) 249-253.

[20] Li, Z., Park, J. B., Chen, G. and Joo, Y. H., Generating chaos via feedback control from a stable TS fuzzy system through a sinusoidal nonlinearity, Int. J. Bifur. Chaos 12 (2002) 2283-2291.

[21] Susko, I. and Gardini, L., Center bifurcation for two-dimensional border-collision normal form, Int. J. Bifur. Chaos 18(4) (2008) 1029-1050.

[22] Albers, D. J., Sprott, J. C. and Crutchfield, J. P., Persistent chaos in high dimensions, Phys. Rev. E 74 (2006) 057201-1-4. 
[23] Alvarez-Llamoza, O., Cosenza, M. G. and Ponce, G. A., Critical behavior of the Lyapunov exponent in type-III intermittency, Chaos Soliton. Fract. 36 (2008) 150156.

[24] Aguirregabiria, J. M., Robust chaos with prescribed natural invariant measure and Lyapunov exponent, (2009) arXiv:0907.3790.

[25] Gallas, J. A. C., The structure of infinite periodic and chaotic hub cascades in phase diagrams of simple autonomous flows, Int. J. Bifur. Chaos 20 (2010) 197-211.

[26] Sogo, K., Inverse problem and central limit theorem in chaotic map theory, J. Phys. Soc. Japan 68 (1999) 3469-3472.

[27] Sogo, K., Inverse problem in chaotic map theory, Chaos Soliton. Fract. 41 (2009) $1817-1822$.

[28] Majumdar, M. and Mitra, T., Robust chaos in dynamic optimization models, Ricerche Economiche 48(3) (1994) 225-240.

[29] Banergee, S., Yorke, J. A. and Grebogi, C., Robust chaos, Phys. Rev. Lett. 80(14) (1998) 3049-3052. 